\title{
Cálculo de un indicador de calidad de vida básico para Bogotá por secciones censales mediante análisis factorial
}

\section{CALCULATING A BASIC QUALITY OF LIFE INDICATOR FOR BOGOTÁ BY CENSUS SECTION USING FACTOR ANALYSIS}

\author{
CÁLCULO DE UM INDICADOR BÁSICO DE QUALIDADE DE VIDA PARA \\ BOGOTÁ POR SEÇÕES CENSITÁRIAS ATRAVÉS DE ANÁLISE FATORIAL
}

Para citar este artículo: Mayorga Henao, J. M., García García, D. M. y

Barrera Gutiérrez, R. A. (2019). Cálculo de un indicador de calidad de vida

básico para Bogotá por secciones censales mediante análisis factorial. Pers-

pectiva Geográfica, 24(1), 53-73. https://doi.org/10.19053/01233769.7861
José Mario Mayorga Henao ${ }^{1}$

Diva Marcela García García ${ }^{2}$

Rafael Andrés Barrera Gutiérrez ${ }^{3}$

\section{Resumen}

El artículo tiene como objetivo calcular y espacializar un indicador de calidad de vida básico por secciones censales para Bogotá, según la información disponible del censo del año 2005. Para ello se utiliza un análisis factorial para establecer un índice que

1 Sociólogo y Doctorando en Geografía de la Universidad Nacional de Colombia. Magíster en Geografía de la Universidad de Chile. Magíster en Planeación Urbana y Regional y Especialista en Derecho Urbanístico de la Pontificia Universidad Javeriana. Especialista en Estadística Aplicada de la Fundación Universitaria los Libertadores.. jmmayorgahenao@gmail.com. https://orcid.org/0000-0002-1039-0407

2 Socióloga, doctoranda en Demografía del Centro de Estudios Demográficos y la Universidad Autónoma de Barcelona. Magíster en Urbanismo de la Universidad Nacional de Colombia. Máster en Estudios Territoriales y de la Población con especialidad en Demografía del Centro de Estudios Demográficos y la Universidad Autónoma de Barcelona. Profesora de la Facultad de Ciencias Sociales de la Pontificia Universidad Javeriana, Bogotá, Colombia. diva.garcia@ javeriana.edu.co. https://orcid.org/0000-0002-4773-6897

3 Economista, Magíster en economía y Doctorando en Estudios Políticos de la Universidad Externado de Colombia.

Profesor de la Facultad de Economía de la Escuela Colombiana de Ingeniería Julio Garavito. Bogotá, Colombia. randresbg@yahoo.com. https://orcid.org/0000-0001-7773-8984 
agregue las variables seleccionadas y un análisis espacial que permita definir si existe una correlación espacial del índice. Lo anterior se enmarca en una reflexión sobre los indicadores para medir la calidad de vida y la importancia de su espacialización en una escala que permita entender patrones de diferenciación de los grupos sociales en el espacio urbano. Como hallazgos, se identificaron agrupaciones de baja calidad de vida que superan el ámbito de la localidad, y, en otros casos, se registró una amplia diversidad al interior de dicha entidad.

Palabras clave: análisis espacial, análisis factorial, calidad de vida.

\section{Abstract}

This article intends to calculate and spatialize a basic quality of life indicator for Bogotá by census section, according to the information available from the 2005 census. To this end, a factor analysis to establish an index that aggregates selected variables and a spatial analysis to define whether there is a spatial correlation of the index are used. This is framed by a reflection on the indicators to measure the quality of life and the importance of their spatialization on a scale that helps to understand differentiation patterns of social groups in urban space. As findings, groups with low quality of life exceeding the scope of locality were identified and, in other cases, wide diversity was reported within such locality.

Keywords: Spatial analysis, factorial analysis, quality of life.

\section{Resumo}

O presente artigo tem como objetivo calcular e espacializar um indicador de qualidade de vida básica por setores censitários para Bogotá, segundo a informação disponível no censo de 2005. Para isso, utiliza-se uma análise fatorial para estabelecer um índice que agrega as variáveis selecionadas e uma análise espacial que permite definir se existe uma correlação espacial do índice. O exposto acima é enquadrado em uma reflexão sobre os indicadores para medir a qualidade de vida e a importância de sua espacialização em uma escala que permita entender padrões de diferenciação dos grupos sociais no espaço urbano. Como descobertas, identificaram-se associações de baixa qualidade de vida que excedem o escopo da localidade, e, em outros casos, registrou-se uma ampla diversidade no interior da referida entidade.

Palavras chave: Análise espacial, análise fatorial, qualidade de vida. 


\section{Introducción}

El estudio de la calidad de vida ha sido reconocido como prioritario tanto por la academia como por los tomadores de decisiones y los ejecutores de políticas públicas en todo el mundo. Sin embargo, la medición del fenómeno es una tarea que plantea numerosos desafíos, empezando por la definición de las posturas conceptuales que se asumirán, y de las que dependen los resultados y los usos potenciales. Desde la definición del concepto de calidad de vida, el debate es amplio y pasa por referencias a las necesidades, los estándares de vida y la insuficiencia de recursos, entre muchos otros (Spicker, 1999).

Para el caso del presente artículo, el concepto del cual se parte es el desarrollado por Sen, quien señala que la "calidad de vida se centra en la forma en que transcurre la vida humana y no solo en los recursos o en la renta que posee un individuo" (Sen, 2004, p. 42). Esta visión abarcadora y compleja plantea que para que las personas puedan tener una calidad de vida digna es necesario que tengan capacidades, las cuales son un paso intermedio que conecta la utilidad con el consumo de bienes. Así, la calidad de vida estaría asociada a la existencia de capacidades del hogar como una función que depende de la disponibilidad y el consumo de bienes (Feres y Mancero, 2001). Cuando se garantiza el acceso a los bienes y los servicios básicos, se genera un efecto sobre la posibilidad de los individuos de desarrollarse con libertad en sus contextos territoriales. Dichas garantías pueden concentrarse y generar situaciones de desigualdad, o pueden generalizarse y producir una espiral que aumenta la calidad de vida, la libertad y las posibilidades de todos los habitantes de la ciudad (Barbosa y González, 2014).
Pero el desafío para la medición de la calidad de vida supera el ámbito conceptual y se traslada al plano de lo metodológico, en donde la disponibilidad de datos y las diferentes posturas ideológicas han llevado a un camino avanzado en el cálculo y el diseño de indicadores de pobreza que permiten aproximarse de alguna manera a la noción de calidad de vida y bienestar humano. Estos identifican a la población con carencias a partir de métodos directos (observación de los bienes físicos y características particulares de los hogares) o indirectos (identificación del nivel de ingreso), por mencionar solo algunas de las clasificaciones existentes (Feres y Mancero, 2001).

Mientras el método directo hace una relación del bienestar con el consumo realizado (para satisfacer una o varias necesidades básicas), el método indirecto se enfoca en la capacidad para realizar dicho consumo. A estos dos métodos se suma aquel que vincula a los dos anteriores. Así, los métodos conocidos para medir la pobreza son el de la línea de pobreza (LP) ${ }^{4}$, el de necesidades básicas insatisfechas (NBI) y el integrado, que los combina (Barneche y et al., 2010). En Colombia se cuenta, entre otros, con "índices multidimensionales de pobreza, calidad de vida y desarrollo humano como el índice de Necesidades Básicas Insatisfechas (NBI), el Índice de Condiciones de Vida (ICV) y el índice de focalización del gasto social SISBEN (en sus versiones I, II y II)" (Angulo Salazar, Díaz Cuervo y Pardo Pinzón, 2011, p. 4) 5 .

4 González (2011) hace un análisis crítico sobre los alcances de este método. Así mismo, indica la importancia de "tener como mira final el enfoque de capacidades $y$, al mismo tiempo, avanzar en la mejora de los indicadores multidimensionales" (p. 101).

5 En este escrito, los autores indican la metodología que se sigue en la construcción del índice, su importancia para Colombia y las posibilidades que tiene para ser usado a nivel municipal. 
Así, Colombia es uno de los países de América Latina, junto con México, pioneros en desarrollar mediciones oficiales de pobreza multidimensional. Desde 2011, el Departamento Nacional de Planeación (DNP), siguiendo la metodología propuesta por Oxford Poverty and Human Development Initiative (Ophdi), usa una metodología compuesta por cinco dimensiones (educación, niñez y juventud, trabajo, salud, y vivienda y servicios públicos) (Santos, 2014; González, 2011). Dicha medición se ha usado para la focalización del gasto, "para monitorear las políticas regionales y para definir objetivos sobre intervenciones específicas" (Santos, 2017, p. 23).

Todo este avance en la medición y la comprensión de la calidad de vida ha conducido a nuevas discusiones, tendientes a revelar cómo el desarrollo, entendido como la ampliación de las capacidades de las personas, tiene connotaciones espaciales (Barbosa y González, 2014). De allí que la incorporación de criterios espaciales al estudio de la calidad de vida sea un aporte reciente a la comprensión del fenómeno, cuyo análisis permite “conocer el papel de diferentes variables del territorio en la dinámica de la pobreza y el grado en que la interacción entre las características sociales y territoriales influyen en la conformación de conglomerados de pobreza o bienestar" (Estrada y Moreno, 2013, p. 208).

Lo anterior se vincula a la comprensión del fenómeno de la calidad de vida desde la vivencia de las personas sobre su territorio, que puede estar dotado o no de infraestructuras de calidad que les permitan la inserción en mercados laborales rentables, de manera que se logren condiciones de movilidad social.

En esta línea, el problema de la escala de los datos resulta crucial tanto para el estudio de diferentes problemáticas en la escala micro como para permitir una mirada de conjunto a nivel de ciudad acerca de fenómenos como la segregación social, que tiene como una de sus dimensiones el grado de concentración espacial de los grupos sociales o, visto desde otra óptica, la homogeneidad social de las áreas internas de la ciudad (Sabatini, 2003). Especial importancia tiene la selección de las unidades espaciales para analizar la distribución de la calidad de vida en un entorno urbano, dado que la investigación social ha mostrado que las medidas no espaciales o con unidades muy agregadas tienden a invisibilizar las condiciones negativas de algunos grupos cuya localización espacial refuerza sus privaciones en términos de calidad de vida (Garroncho y Campos, 2013).

De esta forma, pasar de macroterritorios administrativos a unidades espaciales más ajustadas a la homogeneidad social y urbanística de las ciudades se presenta como un imperativo metodológico para lograr una mejor comprensión del fenómeno.

En Colombia, este enfoque territorial del análisis de la pobreza ha incorporado principalmente como unidad de análisis la escala municipal (Galvis y Meisel, 2010; Vargas Mesa, 2012; Estrada y Moreno, 2013) para identificar tendencias regionales de concentración de la calidad de vida (o su ausencia). Los estudios que analizan escalas intramunicipales han sido escasos y se limitan a las grandes ciudades (Departamento Administrativo de Planeación Municipal de Cali, 2011). Para el caso de Bogotá, también se ha avanzado en la medición de la calidad de vida a nivel intraurbano utilizando principalmente dos escalas en la medición espacial de indicadores: una por localidades y otra por estratos. 
Un ejemplo de los estudios que utilizan datos agregados a nivel de localidad es el desarrollado por la Secretaría Distrital de Planeación (SDP) desde el año 2007, en el cual se elaboran clasificaciones socioeconómicas con base en los resultados de la Encuesta de Calidad de Vida (representativa estadísticamente a nivel local) que agrupan a la población según condiciones altas, medias y bajas a partir de una amplia variedad de indicadores de la vivienda, el hogar y las personas (SDP, 2007). A pesar de la alta utilidad de los hallazgos producidos, cabe subrayar que los 20 macroterritorios administrativos que tiene la ciudad presentan una heterogeneidad social y urbanística bastante amplia, por lo que la unidad de análisis contiene datos altamente dispersos (SDP, 2013).

Por otro lado, se identifican algunas investigaciones que "infieren condiciones en la calidad de vida a partir de los estratos" (Aliaga y Álvarez, 2010). Se trata de un índice que elabora la Administración de la ciudad, en el que se evalúa la calidad de las viviendas y el entorno urbano asignando un número de estrato del 1 al 6 a un grupo de viviendas que, por lo general, corresponde a una manzana (SDP, 2007). Sin embargo, aunque se puede reconocer que los estratos se han constituido en un referente social y de autopercepción para calificar la situación de la población, utilizarlos como fuente para el desarrollo de estudios que permitan establecer las condiciones socioeconómicas presenta un fuerte error metodológico, en tanto es una medición que no se elabora en función de las características de los hogares, sino de las viviendas, elemento que comporta solo uno de los factores a evaluar en la calidad de vida.

Se adolece entonces de indicadores disponibles a baja escala espacial, que permitan capturar con precisión las diferencias de los grupos sociales en el espacio de la ciudad. De hecho, en el caso bogotano no hay investigaciones que tengan en cuenta el problema de la unidad espacial modificable, es decir, que incluyan en sus reflexiones y resultados una discusión sobre la escala de los datos y cómo los resultados pueden variar en función de la desagregación o agrupación espacial de los mismos (Ortiz y Escolano, 2013). Por ello, el objetivo del presente artículo es hacer el cálculo de un indicador de calidad de vida a escala de sector censal para la ciudad de Bogotá e identificar la existencia de tendencias espaciales del fenómeno. Dado el nivel de desagregación territorial, los datos óptimos para esta tarea son los censales en su edición más reciente, la de 2005, que fueron procesados a través del método estadístico de componentes principales, para agregar y ponderar las características en la unidad de sector censal.

\section{Materiales y métodos}

Como ya se dijo, para calcular un indicador de calidad de vida básico (ICVB) por secciones censales, de manera tal que se pueda observar con mayor claridad su tendencia espacial en Bogotá, se recurrió a la información del censo del año 2005 realizado por el Departamento Administrativo Nacional de Estadística (DANE).

En Bogotá se identificaron las áreas geográficas del DANE a partir del reconocimiento del código departamental, el código municipal y la identificación exacta de las secciones censales que se encuentran al interior del ámbito. Una vez identificadas, se procedió a analizar los principales datos para cada una de las áreas geográficas en el sistema de consulta Redatam del DANE. 
Al espacializar la información censal, como se puede observar en la Figura 1, la distribución de hogares en Bogotá tiene una concentración en las secciones periféricas, en las que hay mayor densidad. En contraste, las secciones céntricas presentan concentraciones de población más bajas.

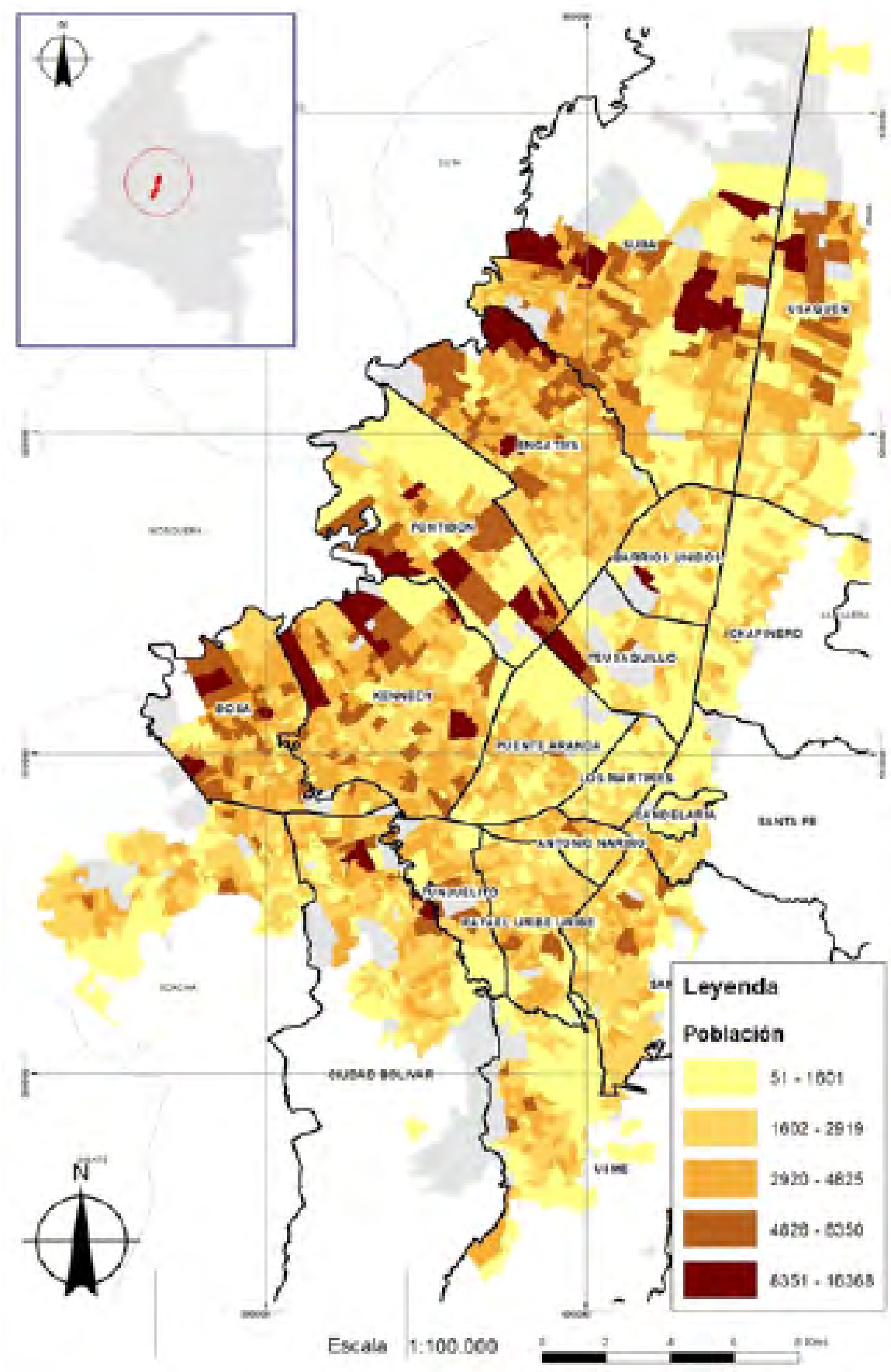

FIGURA 1.

Distribución de hogares por sección censal. Fuente: Elaboración propia con datos del DANE (2005). 
Por su parte, la selección de las variables a incluir en el indicador construido estuvo relacionada con las decisiones teóricas tomadas, así como con la disponibilidad de información en Redatam. Como puede observarse en la Tabla 1, al igual que en Mayorga, García y Hernández (2017), se incluyeron las variables de vivienda y entorno, de dependencia económica y de logro educativo ${ }^{6}$ que evalúa la metodología NBI. Adicionalmente, se incluyó la información de no consumo de alimentos en la úl- tima semana como un indicador de falta de recursos para la alimentación básica, así como el tamaño del hogar como un indicador de vulnerabilidad demográfica, entendido como una desventaja adicional para algunos hogares y grupos de población que contribuye de forma concomitante con otras variables a producir intra e intergeneracionalmente situaciones de riesgos específicos (Centro Latinoamericano y Caribeño de Demografía [Celade], 1999).

6 En este grupo de variables no se incluyó la proporción de personas en edad escolar sin vinculación al sistema educativo, ya que se evaluaron las condiciones de cobertura casi universal de la educación básica en la ciudad.

\section{TABLA 1. DIMENSIONES E INDICADORES O VARIABLES A CONSIDERAR}

\begin{tabular}{ll}
\multicolumn{1}{c}{ Dimensiones } & \multicolumn{1}{c}{ Indicador o variable } \\
\hline Hacinamiento no mitigable & Hogares por vivienda \\
\hline Tipología de vivienda & Tasa de hogares en viviendas según tipo \\
\hline Cobertura de servicios públicos & Tasa de hogares sin servicio de acueducto \\
\hline Tamaño del hogar & Tasa de hogares sin servicio de alcantarillado \\
\hline Actividad desarrollada por los integrantes del hogar & $\begin{array}{l}\text { Tasa de hogares sin servicio de energía } \\
\text { Tasa de población mayor de } 17 \text { años sin empleo }\end{array}$ \\
\hline $\begin{array}{l}\text { No consumo de alimentos en la última semana } \\
\text { hogar }\end{array}$ & $\begin{array}{l}\text { Tasa de personas que no consumieron ninguna de las tres comidas, algún día } \\
\text { de la última semana }\end{array}$ \\
\hline
\end{tabular}

Fuente: Mayorga, García y Hernández (2017). 
Los indicadores fueron homogenizados en una escala de 1 a 100 mediante el procedimiento descrito en la ecuación 1. Para homogenizar las variables se asumen un valor máximo y un valor mínimo para cada indicador, según sea un indicador de escala negativa o positiva:

$$
\begin{aligned}
& I H_{j}=\left[\frac{I_{j}-\operatorname{Max}(I)}{\operatorname{Max}(I)-\operatorname{Min}(I)}\right] * 100 *(-1) \\
& I H_{j}=\left[\frac{I_{j}-\operatorname{Min}(I)}{\operatorname{Max}(I)-\operatorname{Min}(I)}\right] * 100
\end{aligned}
$$

(Ec. 1): modelo de estandarización de indicadores con escala negativa (elaboración propia)

(Ec. 2): modelo de estandarización de indicadores con escala positiva (elaboración propia)

Una vez estandarizados los indicadores, se procedió a utilizar dos procedimientos estadísticos. En primer lugar, se realizó un análisis factorial para producir un índice compuesto que contenga el peso de cada indicador en el ICVB. Posteriormente, para establecer la tendencia espacial del ICVB en Bogotá y definir si tiene un comportamiento tipo clúster, se utilizó el índice de Moran, que mide la autocorrelación espacial basada en las ubicaciones y los valores de las unidades censales simultáneamente. Los dos procedimientos se describen a continuación.

\subsection{Análisis factorial}

El análisis factorial es un método inferencial que forma parte de las técnicas estadísticas multivariantes. Se usa para encontrar agrupaciones de variables, de forma tal que las variables dentro de cada grupo se encuentren altamente correlacionadas y los grupos estén relativamente intercorrelacionados. De esta manera es posible efectuar la reducción de datos que permite encontrar grupos de variables con un significado o marca común (Montoya Suárez, 2007; Méndez Martínez y Rondón Sepúlveda, 2012). En este sentido, “los mé- todos factoriales [...] producen resultados más o menos interpretables [que] no son una simple descripción, sino que ponen de manifiesto la estructura existente entre los datos" (Abascal Fernández y Landaluce Calvo, 2002, p. 110).

Dicho método busca realizar "un análisis de la estructura subyacente en una serie de variables" (Morales Vallejo, 2013, p. 3) o "describir, si es posible, las relaciones entre varias variables en términos de pocas variables no observables, pero importantes, llamadas Factores" (Johnson, 2007, p. 481), de modo que expliquen una buena parte de la variabilidad de las varianzas o la mayor cantidad de información que se encuentra en los datos iniciales.

Existen dos tipos de análisis factorial: exploratorio y confirmatorio. En el primero se establece la contribución de las variables originales a cada uno de estos nuevos factores y se espera que el investigador no tenga conocimiento a priori de la nueva estructura, o, si tiene alguna idea, que esta sea menor; en el segundo escenario se eliminan del análisis aquellas variables que sean poco relevantes o que tengan mucha colinealidad con otras varia- 
bles. En el segundo, se supone previo el número de factores, la relación entre los mismos y la relación de estos con las variables; también se pueden formular y probar hipótesis mucho más concretas y específicas, "aunque en ciencias sociales no es fácil especificar de manera precisa el valor de las correlaciones con cada factor" (Morales Vallejo, 2013, p. 10).

Ahora bien, para que el análisis factorial tenga sentido debe cumplir dos características básicas: parsimonia e interpretabilidad. La primera indica que los fenómenos deben explicarse con la menor cantidad de elementos, por lo que el número de factores debe ser el menor posible. La segunda indica que una solución factorial considerada buena es aquella que resulta de fácil interpretación y es relativamente sencilla; también se relaciona con una de las limitaciones de esta técnica: el nombramiento de los factores, que puede resultar problemático porque los nombres de los factores pueden no reflejar de manera precisa las variables dentro del factor (Yong y Pierce, 2013).

Los pasos a seguir para realizar el análisis factorial, según Morales Vallejo (2013), Montoya Suárez (2007) y Yong y Pierce (2013), son:

1. Elaboración de la matriz de correlaciones ${ }^{7}$ : se busca realizar un cálculo de una matriz capaz de expresas la variabilidad conjunta de las variables.

2. Extracción de los factores comunes.

7 “En el análisis factorial es costumbre empezar con una matriz de correlaciones entre variables y terminar con una matriz de pesos factoriales, que pueden interpretarse [...] como correlaciones entre las variables y ciertas construcciones hipotéticas Ilamadas 'factores'” (Comrey, 1985, p. 35).
3. Rotación de los factores iniciales: ayuda a clarificar la estructura subyacente a las variables y pretende encontrar una estructura más simple y de más fácil interpretación.

4. Denominación a los factores encontrados.

En el modelo matemático, el análisis de factores clásico, denota el número de variables $\left(X_{1}, X_{2}, X_{3}\right.$, ..., $\left.X_{p}\right)$ y denota el número de factores subyacentes $\left(F_{1}, F_{2}, \ldots, F_{m}\right) . X_{j}$ denota la variable representada en los factores latentes. El modelo supone que hay $m$ factores subyacentes en los cuales cada variable observada es una función lineal de estos factores junto con un valor residual. Este modelo intenta reproducir las correlaciones máximas.

$$
\begin{aligned}
& X_{1}=a_{11} F_{1}+a_{12} F_{2}+\cdots \cdots+a_{1 m} F_{m}+e_{1} \\
& X_{2}=a_{21} F_{1}+a_{22} F_{2}+\cdots \cdots+a_{2 m} F_{m}+e_{2} \\
& \cdots \cdots \cdots \cdots \cdots \cdots \cdots \cdots \cdots \cdots \cdots \cdots \cdots \cdots \cdots \cdots \cdots \cdots \cdots \cdots \cdots \cdots \cdots \cdots \cdots \cdots \cdots \\
& X_{p}=a_{p 1} F_{1}+a_{p 2} F_{2}+\cdots \cdots+a_{p m} F_{m}+e_{p}
\end{aligned}
$$

Matricialmente se puede expresar como $\mathrm{X}=\mathrm{Af}+$ E, donde:

$\mathrm{x}=$ Vector de las variables originales.

A = Matriz factorial. Recoge las cargas factoriales o saturaciones.

$\mathrm{f}=$ Vector de factores comunes.

$\mathrm{E}=$ Vector de factores únicos o específicos.

El estadístico básico usado en el análisis factorial es el coeficiente de correlación que determina la relación entre dos variables. Se examina si las variables tienen alguna característica en común y luego 
se obtiene una correlación o matriz de covarianzas (Yong y Pierce, 2013).

Los factores comunes y los específicos son variables hipotéticas y se supondrá para el análisis:

1. Los factores comunes son variables con media cero y varianza 1, que se encuentran correlacionados $\left(E\left(F_{i}\right)=0 ; \operatorname{Var}\left(F_{i}\right)=1\right.$.

2. Los factores específicos son variables con media cero $\left(E\left(e_{i}\right)=0\right)$, con varianzas que pueden diferir y covarianza cero $\left(\operatorname{Cov}\left(e_{i}, e_{j}\right)=0\right.$ si $i \neq j$; con $j, i=1,2, \ldots, p)$. Se presenta el supuesto de que hay incorrelación entre sí (Cov $\left.\left(F_{i}, e_{j}\right)=0 ; \forall_{i}=1, \ldots, m ; j=1, \ldots, p\right)$, pues de otra manera los factores comunes tendrían la información.

3. Los factores comunes y los factores únicos están incorrelacionados entre sí, lo que permite realizar inferencias que posibilitan distinguir entre los factores comunes y los específicos.

Con base en el modelo y en las hipótesis formuladas, se encuentra que la varianza, en cada variable, según la ecuación 4:

$$
\sigma_{i}^{2}=\sum_{j=1}^{m} \sigma_{i j}^{2}+\vartheta_{i}
$$

$\sigma_{i}^{2}=\sum_{j=1}^{m} \sigma_{i j}^{2}+\vartheta_{i} \quad$ Siendo $\vartheta_{i}$ la varianza de $e_{i}$, se puede descomponer en (Montoya, 2007, p. 282):

(1) Variabilidad explicada por una serie de factores comunes con el resto de variables, que será llamada comunalidad de la variable.

$$
h_{i}^{2}=\sum_{j=1}^{m} \sigma_{i j}^{2}
$$

(Ec. 5)

La comunalidad de la variable $X_{i}$ representa la proporción de la varianza que los distintos factores, en su conjunto, explican de la variable. Se encuentra entre 0 (cuando los factores no dan explicación de la variable) y 1 (si los factores explican el 100\% de la variable).

(2) La parte de la variabilidad que es propia a cada variable, que no es común con el resto de variables. A esta se le denomina varianza especifica o especificidad de la variable.

\section{2. Índice de Moran}

En el estudio de patrones y procesos espaciales, la noción de autocorrelación espacial se vincula con la idea de que valores observados en áreas cercanas o adyacentes serán más similares que los esperados bajo el supuesto de independencia espacial o de aquellos que se encuentran más lejanos (Giraldo Henao, 2011; Paradis, 2015).

Los índices globales contrastan la hipótesis de autocorrelación espacial en la totalidad del área de estudio, pero no permiten determinar si el esquema de autocorrelación espacial identificado se presenta también a nivel local. De todos los índices globales el más utilizado es el I de Moran (Sánchez Rivero, 2008). La fórmula del coeficiente I de Moran es: 
$I=\frac{n}{S_{0}} \frac{\sum_{i=1}^{n} \ldots: \vdots \sum_{j=1}^{n} w_{i, j} z_{i} z_{j}}{\sum_{i=1}^{n} z_{i}^{2}}$

En donde $\mathrm{n}$ significa el número de las unidades (áreas o puntos) en el mapa,Wij es la matriz de distancias que define si las áreas o puntos geográficos, i y j, son vecinos o no. Zi es la desviación de un atributo del elemento i de su media (xi -'x), y es el agregado de todas distancias espaciales (Vilalta y Perdomo, 2005).

$$
S_{0}=\sum_{i=1}^{n} \sum_{j=1}^{n} w_{i, j}
$$

(Ec. 7)

\section{Resultados}

\subsection{Análisis factorial}

En el análisis factorial, con las variables seleccionadas, se obtuvo un KMO de 0,736, el cual es considerado alto y permite una aceptable adecuación de los factores en el modelo. Igualmente, se comparó la prueba de esfericidad de Bartlett, que con un $P$ valor de $(0,000)<0,05$ permite aceptar que el modelo es válido.

\section{TABLA 2. PRUEBAS DE VALIDEZ DEL MODELO}

\begin{tabular}{cc}
\hline gl & 45 \\
\hline Sig. & 0,000 \\
\hline
\end{tabular}

Fuente: Elaboración propia.

Al revisar las correlaciones entre las variables fue necesario excluir tipo de vivienda cuarto, dado que no se correlacionaba con, por lo menos, el $70 \%$ de las demás. Como se muestra en la Tabla
3, al aplicar el análisis factorial, las diez variables incluidas se reducen a tres factores que explican el $65 \%$ de la varianza, por lo cual se refuerza la validez del modelo. 


\section{TABLA 3. VARIANZA TOTAL EXPLICADA}

\begin{tabular}{|c|c|c|c|c|c|c|c|c|c|}
\hline \multirow{2}{*}{ 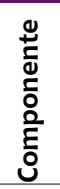 } & \multicolumn{3}{|c|}{ Autovalores iniciales } & \multicolumn{3}{|c|}{$\begin{array}{l}\text { Sumas de las saturaciones al cua- } \\
\text { drado de la extracción }\end{array}$} & \multicolumn{3}{|c|}{$\begin{array}{l}\text { Suma de las saturaciones al cuadrado } \\
\text { de la rotación }\end{array}$} \\
\hline & Total & $\begin{array}{l}\% \text { de la va- } \\
\text { rianza }\end{array}$ & $\%$ acumulado & Total & $\begin{array}{l}\% \text { de la } \\
\text { varianza }\end{array}$ & $\%$ acumulado & Total & $\begin{array}{l}\% \text { de la } \\
\text { varianza }\end{array}$ & $\%$ acumulado \\
\hline 1 & 3,599 & 35,990 & 35,990 & 3,599 & 35,990 & 35,990 & 2,803 & 28,034 & 28,034 \\
\hline 2 & 1,647 & 16,470 & 52,460 & 1,647 & 16,470 & 52,460 & 2,123 & 21,228 & 49,261 \\
\hline 3 & 1,324 & 13,241 & 65,700 & 1,324 & 13,241 & 65,700 & 1,644 & 16,439 & 65,700 \\
\hline 4 & 0,879 & 8,791 & 74,491 & & & & & & \\
\hline 5 & 0,714 & 7,140 & 81,632 & & & & & & \\
\hline 6 & 0,624 & 6,240 & 87,872 & & & & & & \\
\hline 7 & 0,495 & 4,955 & 92,827 & & & & & & \\
\hline 8 & 0,330 & 3,297 & 96,124 & & & & & & \\
\hline 9 & 0,253 & 2,530 & 98,654 & & & & & & \\
\hline 10 & 0,135 & 1,346 & 100,000 & & & & & & \\
\hline
\end{tabular}

Método de extracción: análisis de componentes principales.

Fuente: Elaboración propia

Para mejorar las correlaciones entre los factores y las variables se aplicó un proceso de rotación varimax, el cual permite definir mejor la relación entre los elementos y la denominación conceptual de los factores. Se obtuvo que el factor 1 explica el $28 \%$, el factor 2 explica el $21 \%$ y el factor 3 el 16\% de la varianza total de las variables. De tal forma, se puede afirmar que es un modelo equilibrado en el peso de los factores, dado que ninguno representa el doble del porcentaje de varianza.
Con respecto a las comunalidades, se identifica que en todas las variables hay una extracción de más del $50 \%$ en los factores. Las variables que mejor se explican en los factores son: el porcentaje de personas sin educación, el porcentaje de viviendas sin alcantarillado y el porcentaje de viviendas sin energía. Las variables que tienen menor explicación son la cantidad de personas por hogar, el porcentaje de personas sin empleo y el índice de dependencia. 


\section{TABLA 4. COMUNALIDADES}

\begin{tabular}{lcc}
\hline & Inicial & Extracción \\
\hline Personas por hogar & 1.000 & 0,500 \\
\hline Hogares por vivienda & 1.000 & 0,592 \\
\hline Dependencia & 1.000 & 0,579 \\
\hline Dependencia económica & 1.000 & 0,720 \\
\hline Porcentaje de personas con ayuno & 1.000 & 0,779 \\
\hline Porcentaje de personas sin educación & 1.000 & 0,817 \\
\hline Porcentaje de personas sin empleo & 1.000 & 0,815 \\
\hline Porcentaje de viviendas sin energía & 1.000 & 1.000 \\
\hline Porcentaje de viviendas sin alcantarillado & 1.000 & 0,817 \\
\hline Promedio de años de educación alcanzado & 0,697
\end{tabular}

Fuente: Elaboración propia

A partir de las correlaciones de cada variable con los factores (Tabla 5) se obtiene una definición de los factores.

El factor 1 se compone de características de las viviendas especialmente referidas a los servicios públicos, ya que está definido por el porcentaje de viviendas sin energía eléctrica, el porcentaje de viviendas sin servicio de acueducto y el porcentaje de viviendas sin servicio de alcantarillado. De tal forma, teniendo en cuenta las características de las variables, se denominó factor de servicios públicos en la vivienda.

El factor 2 se compone por las variables: número de hogares por vivienda, número de personas por hogar y dependencia económica. A partir de estas variables se denominó factor de composición demográfica, dado que se compone de características de la población con respecto a su estructura de edades, la composición del hogar y la distribución de viviendas en los hogares.

El factor 3 se denominó factor socioeconómico, dado que se compone de características de los individuos y su inserción en la estructura económica. Sus componentes principales son: el no consumo de alimentos en la última semana, el promedio de años de educación de la población, el porcentaje de población sin educación y el porcentaje de población desempleada. 


\section{TABLA 5. MATRIZ DE COMPONENTES ROTADOS}

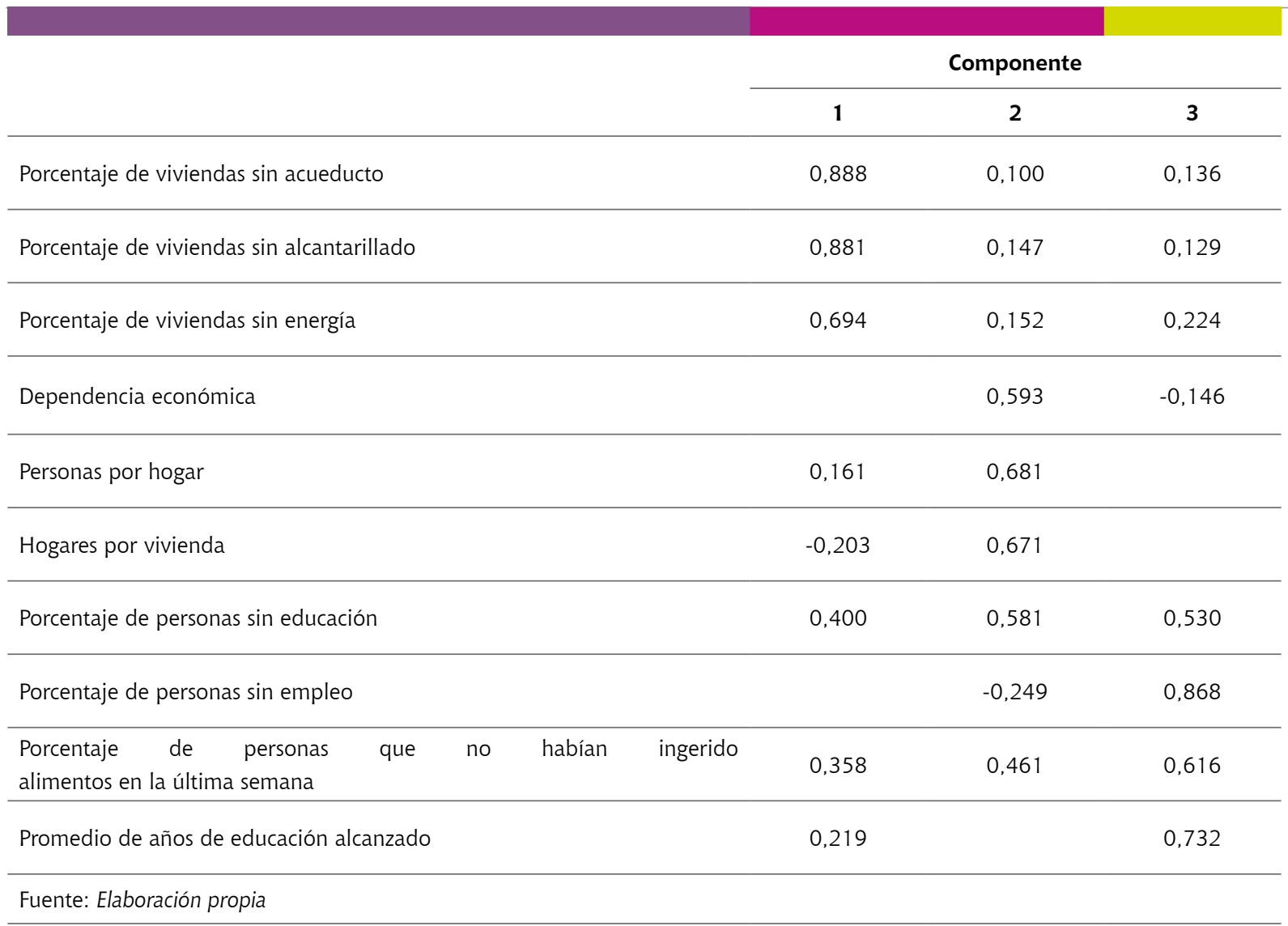

Con base en las puntuaciones factoriales que estiman el valor de cada factor para cada sección mediante el método de regresión, se producen valores con media de 0 y una varianza igual al cuadrado de la correlación múltiple entre las puntuaciones factoriales estimadas y los valores factoriales verdaderos. Por último, se obtuvo un índice, concebido como un promedio en el que cada factor tiene el mismo peso (Mayorga, García y Hernández, 2017).

$$
I C V=\frac{F 1+F 2+F 3}{3}
$$

F1: Servicios públicos en la vivienda

F2: Composición demográfica

F3: Características socioeconómicas

\subsection{Espacialización e índice de}

Moran

Al espacializar los resultados del ICVB por secciones censales en Bogotá, se puede identificar un patrón de diferenciación social en el espacio que indica la concentración de grupos socioeconómicos altos en una cuña de alta renta y grupos socioeconómicos bajos en las periferias de la ciudad. 


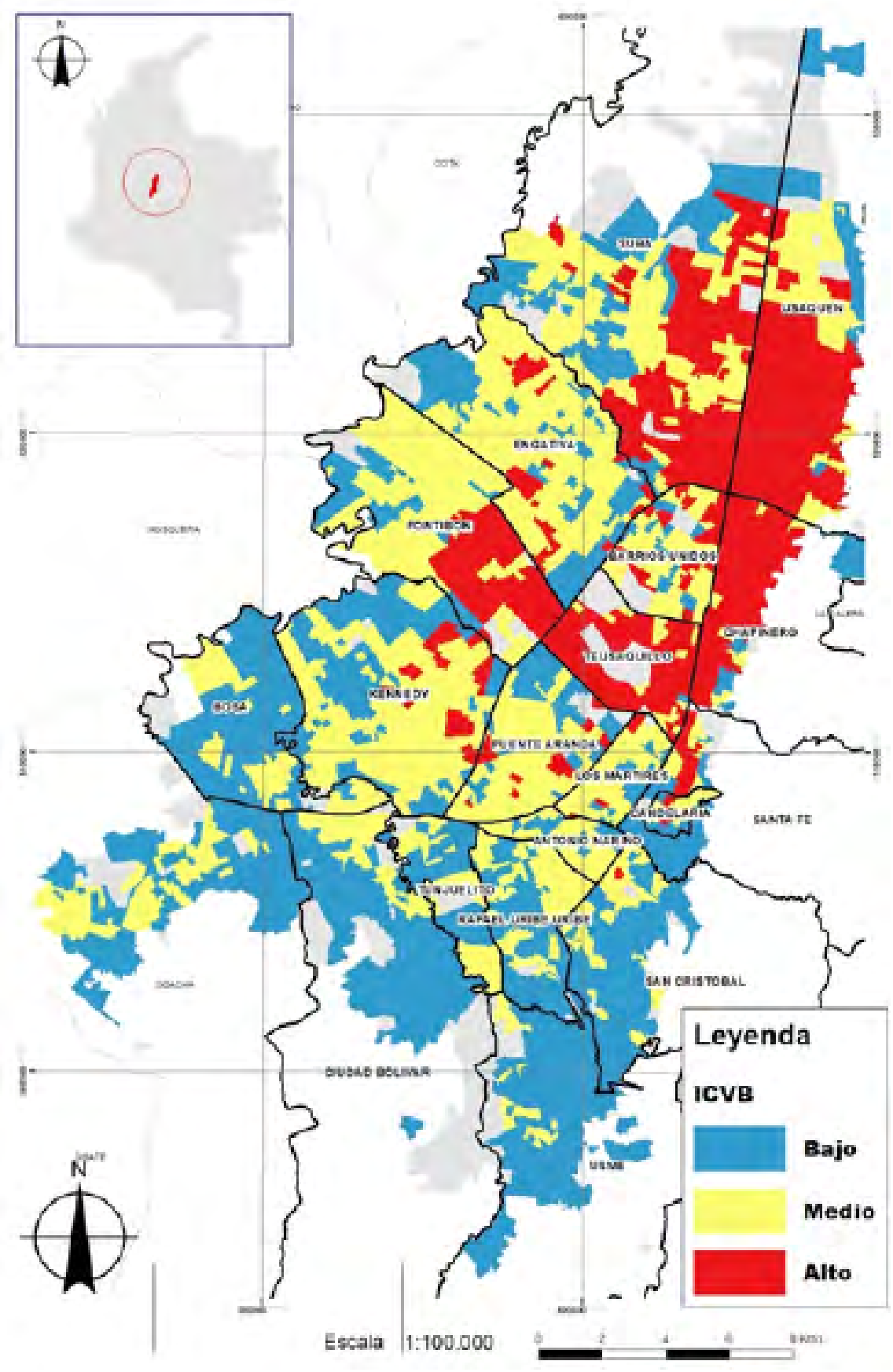

\section{FIGURA 2.}

Espacialización del ICVB por secciones censales, Bogotá, año 2005.

Fuente: Elaboración propia.
De manera descriptiva, se observa que las condiciones más bajas de calidad de vida predominan en los bordes del sur de la ciudad conformando una especie de U que inicia en el oriente y termina en el occidente, con una mayor extensión hacia el sur, en el municipio de Soacha. Las localidades de San Cristóbal, Usme, Rafael Uribe, Tunjuelito, Ciudad Bolívar y Bosa se caracterizan porque más del $80 \%$ de su territorio se encuentra en condiciones de vida bajas. 
La distribución de calidad de vida alta tiene una tendencia de conformación de dos cuñas, una que inicia en el centro, con dirección hacia el norte, y otra que igualmente parte del centro en dirección al occidente. Estas secciones están altamente concentradas y presentan una tendencia espacial clara, que tiende a distanciarse de las secciones de calidad de vida baja por medio de un "colchón" que corresponde a las secciones de calidad de vida media. Se identifican algunas localidades en las que se concentran los valores altos, principalmente en Santa Fe, Teusaquillo, Chapinero y Usaquén.

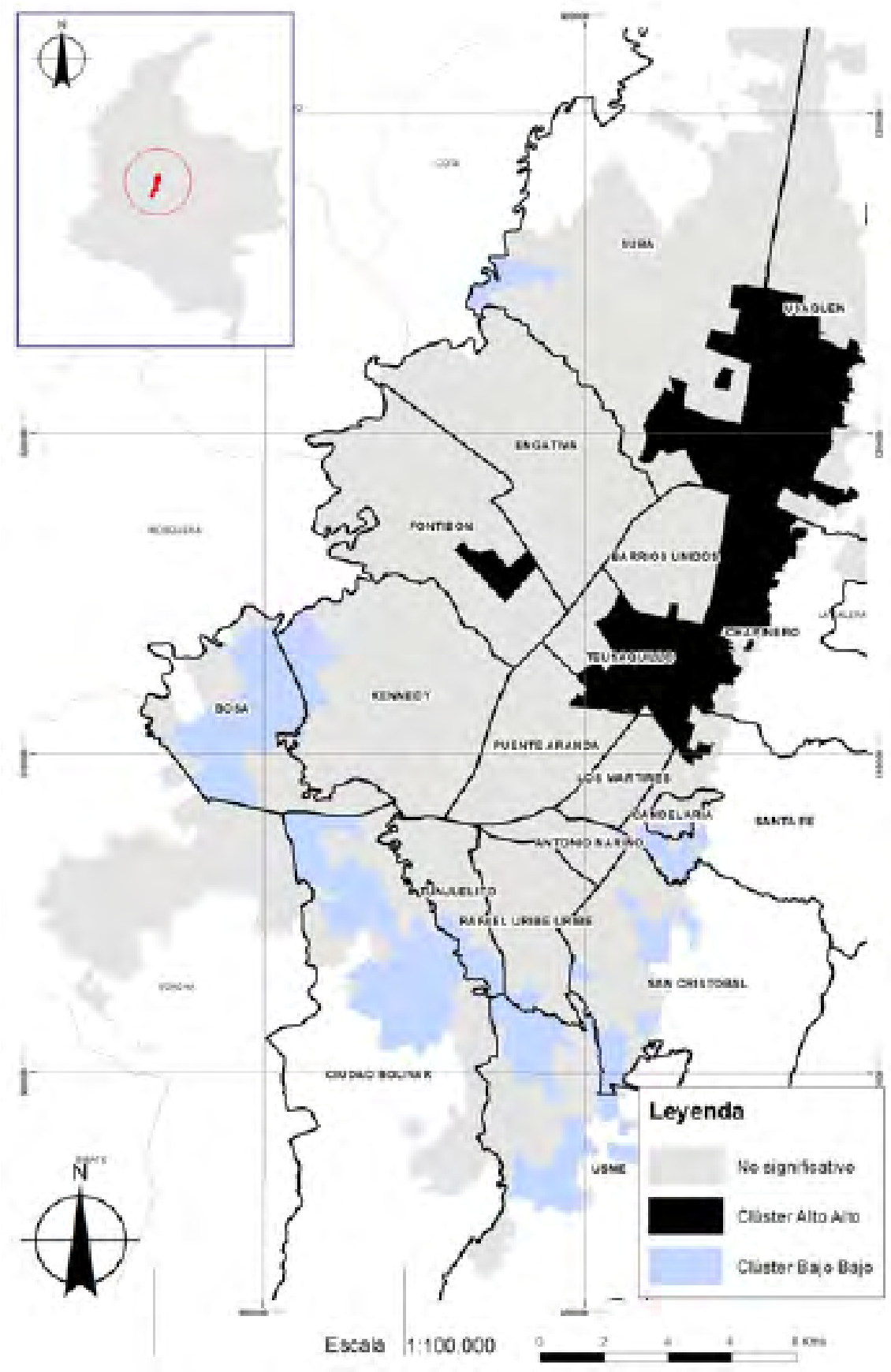

\section{FIGURA 3.}

Índice de Moran y

clústeres espaciales para

el ICV en Bogotá por secciones censales, año 2005.

Fuente: Elaboración propia. 
Al aplicar el índice de Moran con el ICV para las secciones censales de Bogotá, se obtiene que con un $P$ valor menor a un nivel de significancia de 0,05 se acepta la hipótesis de que los valores presentan autocorrelación espacial y se agrupan en clústeres. Al aplicar el índice de Moran y generar los clústeres espaciales, con un $P$ valor de 1,6 * $10^{-8}$, se obtiene una tendencia clara de índices altos en dos ejes que van desde el centro hacia el norte y el occidente de la ciudad. Igualmente, se obtienen unos índices bajos hacia el sur de la ciudad, en un eje que limita la periferia de oriente a occidente.

Espacialmente, se agrupan en el grupo alto-alto aquellas secciones que tienen un valor de ICV por encima de 90 y se encuentran correlacionados espacialmente con sus vecinos. Por el contrario, se asignan a la categoria bajo-bajo aquellos que tienen un ICV por debajo de 85 y se encuentran correlacionados espacialmente con sus vecinos.

\section{Conclusiones}

La medición de indicadores de calidad de vida a una escala territorial intermedia entre la dispersión de los datos por manzanas censales y su agrupación en grandes territorios como las localidades permite hacer una lectura de sus patrones espaciales en la ciudad. De tal forma, representa un avance con respecto a las mediciones que se han hecho en Bogotá con base en la división de las localidades. A diferencia de las investigaciones oficiales en las que se han utilizadado las localidades como unidades espaciales de analisis, en la presente investigación se alcanzó una medición que permite identificar los grados de concentración de grupos con condiciones socioeconómicas homogéneas y su distancia espacial con respec- to a otros grupos. De tal forma, se evidenció que en Bogotá el comportamiento de las condiciones de calidad de vida está altamente sectorizado, en una escala macro generada por la yuxtaposición de secciones micro.

La inclusión de la variable territorial en el estudio de la pobreza, en el caso de Bogotá, confirmó una doble tendencia de la segregación constituida a partir de los esquemas y modos de urbanización. De un lado, tenemos la consolidación de polos de "similares", es decir, grandes áreas en las que solo es posible encontrar población con un estatus socioeconómico alto o bajo según el caso; a partir del índice de Moran, se hace evidente una clusterización más ampliada de clases altas en la zona nororiental de la ciudad. Mientras tanto, las agrupaciones de pobres rodeados de pobres parecen reducirse a las zonas periféricas que conforman el borde de la ciudad. Esta consolidación de extremos socioeconómicos en el territorio evidencia la dificultad para el contacto cara a cara entre "diversos" en el territorio y prende las alarmas con respecto a fenómenos como la estigmatización y la poca capacidad de ascenso social a partir de la construcción de capital social entre personas con diferentes niveles de calidad de vida.

De otra parte, sobresalió el fenómeno de la heterogeidad social en localidades como Usaquén y Suba, que presentan contrastes entre vecinos, lo cual plantea la ventaja de la interacción entre los diversos, pero también señala la dificultad metodológica de tomar la localidad como unidad de análisis representativa para la política pública. La presencia de grupos tan diversos hace que en estas localidades las cifras agregadas resulten dudosas, y que se requiera una planeación diferenciada de bienes y servicios urbanos y sociales. 
En términos metodológicos, cabe señalar que si bien no hay una única manera de agregación de datos (que depende del método de extracción y de rotación), el análisis factorial permitió un acercamiento inicial al panorama que presenta el área de estudio. Adicionalmente, a pesar de las caracterís- ticas generales complejas que se intentan agrupar, los resultados arrojados en este caso facilitaron la nominación de los factores, lo cual va de la mano con los supuestos de parsimonia e interpretabilidad del método utilizado. 


\section{Referencias}

Abascal Fernández, E. y Landaluce Calvo, Ma . I. (2002). Análisis factorial múltiple como técnica de estudio de la estabilidad de los resultados de un análisis de componentes principales. Qüestiió: Quaderns d'estadística i investigació operativa, 26(1-2), 109-122.

Aliaga, L. y Álvarez, M. (2010). Segregación residencial en Bogotá a través del tiempo y diferentes escalas. [Documento de trabajo]. Bogotá: Lincoln Institute of Land Policy.

Angulo Salazar, R. C., Díaz Cuervo, Y. y Pardo Pinzón, R. (2011). Índice de Pobreza Multidimensional para Colombia (IPM.Colombia) 1997-2010. Archivos de Economía n 382. Bogotá: Departamento Nacional de Planeación.

Barbosa W. G. y González, J. I. (2014). Calidad de vida urbana: una propuesta para su evaluación. Revista de Estudios Sociales, 49, 159-175.

Barneche, P., Bugallo, A., Ferrea, H., Ilarregui, M., Monterde, C., Pérez, M. V. y Santa María, T. (2010). Métodos de medición de la pobreza. Conceptos y aplicaciones en América Latina. Entrelíneas de la Política Económica, 26(4), 31-41.

Centro Latinoamericano y Caribeño de Demografía (Celade). (1999). Vulnerabilidad demográfica y desventajas sociales: el caso de Chile. LC/DEM/R.299, Santiago de Chile: Celade.

Comrey, A. (1985). Manual de análisis factorial. Jaime Cabrebra (trad.). Madrid: Cátedra.

Departamento Administrativo Nacional de Estadística (DANE) (2005). Datos Censales Redatam.

Departamento Administrativo de Planeación Municipal de Cali (2011). Pobreza y exclusión social en Cali: un análisis de los hogares y la población de sectores populares y clases medias bajas a través del Siisas 2009. Cali: Alcaldía de Santiago de Cali.

Estrada, L. y Moreno, S. L. (2013). Análisis espacial de la pobreza multidimensional en Colombia a partir del Censo de Población de 2005. Revista IB Dane, 3(1), s. p.

Feres, C. y Mancero, X. (2001). Enfoques para la medición de la pobreza: breve revisión de literatura. Serie Estudios Estadísticos y Prospectivos nº 4. Santiago: Cepal.

Galvis, L. A. y Meisel, A. (2010). Persistencia de las desigualdades regionales en Colombia: un análisis espacial. Documentos de Trabajo sobre Economía Regional y Urbana ${ }^{\circ}$ 120. Cartagena: Banco de la República.

Garroncho, C. y Campos-Alanis, J. (2013). Réquiem por los indicadores no espaciales de segregación residencial. Papeles de Población, 19(77), 269-300.

Giraldo Henao, R. (2011). Estadística espacial, notas de clase. Bogotá: Universidad Nacional de Colombia.

González, J. I. (2011). Utilitarismo y mediciones de pobreza. Revista de Economía Institucional, 13(25), 89-103. 
Johnson, R. A. (2007). Applied multivariate statistical analysis. New Jersey: Pearson Prentice Hall.

Mayorga, J. M., García-García, D. M. y Hernández, L. (2017). Calidad de vida y su correlación con los precios del suelo: aproximación a la segregación residencial en Bogotá. Cuadernos de Vivienda y Urbanismo, 10(19), 22-40. https://doi. org/10.11144/Javeriana.cvu10-19.cvcp

Méndez Martínez, C. y Rondón Sepúlveda, M. A. (2012). Introducción al análisis factorial exploratorio. Revista Colombiana de Psiquiatría, 41(1), 197-207.

Montoya Suarez, O. (2007). Aplicación del análisis factorial a la investigación de mercados. Caso de estudio. Scientia et Technica, 13(35), 281-286.

Morales Vallejo, P. (2013). El análisis factorial en la construcción e interpretación de tests, escalas y cuestionarios. Madrid: Universidad Pontificia Comillas. Recyuperado de http://www.upcomillas.es/personal/peter/investigacion/AnalisisFactorial.pdf.

Ortiz, J. y Escolano, S. (2013). Movilidad residencial del sector de renta alta del Gran Santiago (Chile): hacia el aumento de la complejidad de los patrones socio espaciales de segregación. EURE (Santiago), 39(118), 77-96. http://dx.doi. org/10.4067/S0250-71612013000300004

Paradis, E. (2015). Moran's Autocorrelation Coefficient in Comparative Methods. Recuperado de https://cran.r-project.org/web/packages/ape/vignettes/Moranl.pdf.

Sabatini, F. (2003). La segregación social del espacio en las ciudades de América Latina. Washington, D. C.: BID. Recuperado de https://publications.iadb.org/es/ publicacion/15146/la-segregacion-social-del-espacio-en-las-ciudades-de-americalatina

Sánchez Rivero, M. (2008). Análisis espacial de datos y turismo: nuevas técnicas para el análisis turístico. Una aplicación a caso extremeño. Revista de Estudios Empresariales. Segunda época, 2, 48-66.

Santos, M. E. (2017). Brief history of measurement of multidimensional poverty in Latin America. Dimensions, 2, 18-23.

Santos, M. E. (2014). Measuring multidimensional poverty in Latin America: Previous Experience and the way forward. Oxford: Oxford Poverty \& Human Development Inivitative.

Secretaría Distrital de Planeación (SDP). (2007). Encuesta de Calidad de Vida Bogotá 2007. Resultados preliminares.

Secretaría Distrital de Planeación (SDP). (2013). Índice de prosperidad en Bogotá D.C. Boletines de Ciudad, 49. Recuperado de http://www.sdp.gov.co/sites/default/files/ dice133-boletinindiceprosperidad-2013.pdf

Sen, A. (2004). Desarrollo y libertad. Bogotá: Planeta.

Spicker, P. (1999). Definitions of poverty: eleven clusters of meaning. En D. Gordon, S. Alvarez y P. Spicker. Poverty: An International Glossary (pp. 229-243). Londres: Zed Books. 
Vargas Mesa, O. M. (2012). Aproximación espacial al estudio de la pobreza en Colombia: un estudio aplicado con información del año 2005. (Tesis de grado). Universidad del Valle, Facultad de Ciencias Sociales y Económicas, Cali, Colombia. Recuperado de http://bibliotecadigital.univalle.edu.co/bitstream/10893/3690/4/CB-0449663.pdf

Vilalta y Perdomo, C. (2005). Cómo enseñar autocorrelación espacial. Economía, Sociedad y Territorio, 5(18), 323-333.

Yong, A. G. y Pearce, S. (2013). A beginner's guide to factor analysis: Focusing on exploratory factor analysis. Tutorials in quantitative methods for psychology, 9(2), 79-94. 